\title{
O processamento cognitivo das informações espaciais na navegação digital
}

\author{
The cognitive processing of information in navigation systems
}

Aline de Camargo Barros; Gabriela Botelho Mager

informação espacial, cidades, navegação digital, psicologia cognitiva

Ao longo dos últimos anos, os sistemas de informação para pedestres vêm migrando para o meio virtual. Os aplicativos de navegação digital em dispositivos móveis se estabeleceram como uma ferramenta para a leitura do espaço, orientação e deslocamento na cidade, e tendem a se aprimorarem cada vez mais diante dos avanços tecnológicos. O presente artigo baseia-se em uma análise do processamento cerebral das diversas camadas de informação espacial no meio digital, revelando uma terceirização da memória com desdobramentos no caminhar urbano.

spacial information, cities, navigation system, cognitive psychology

Over the past years, information systems to pedestrians are being introducing in digital media. Navigation systems on mobile devices have become a valuable tool to read the space as to orient and move people around the city, and they tend to improve according to the technological advances. In this context, the present article analyses the brain processing of different information layers in the digital environment, revealing a dependent relationship related to the memory and cognition, which directly influence the urban walkability.

\section{Introdução}

Mapas, setas, signos e pictogramas compõem um sistema de informação do espaço construído que comunica e informa sobre o posicionamento de construções, conexões e limites do território. Quando direcionadas aos pedestres que caminham pela cidade, essas informações alcançam um significado ainda mais relevante, de orientadoras no espaço público e catalisadoras de uma mobilidade ativa.

Ao longo dos últimos anos, com o movimento de digitalização, o desenvolvimento das Tecnologias de Informação e Comunicação e a crescente captação de dados, as informações do espaço migraram para a dimensão virtual, em aplicativos de navegação digital de dispositivos móveis. Um único dispositivo portátil tornou-se capaz de armazenar uma grama de informações variadas sobre o território, que buscam um aprimoramento do conteúdo diante das inovações tecnológicas. Curitiba | Brazil | 2021 
O presente artigo analisa a digitalização dos sistemas de informação para pedestres e analisa os seus efeitos no processamento cognitivo dessas mensagens, agora inseridas no meio digital. Afinal, as informações espaciais da navegação digital em dispositivos móveis auxiliam na orientação espacial ou provocam a desorientação ao terceirizar a memorização da configuração do espaço construído?

\section{A digitalização das informações espaciais}

As mais recentes revoluções tecnológicas da digitalização de dados e do desenvolvimento de Tecnologias de Informação e Comunicação (TIC) nas cidades digitais anunciaram uma nova extensão do corpo humano: os dispositivos móveis. Estes dispositivos atuam como uma interface mediadora na relação interdependente da dimensão cultural do homem e seu território (Hall, 2005), ao fornecerem os mais variados tipos de informações para a expansão do indivíduo como ser social em cidades que se difundem entre o físico e o digital (Ascott, 1995; Lemos, 2010).

Na microescala da rua, este contexto digital amplificou as possibilidades de leitura das metrópoles contemporâneas durante o deslocamento a pé. O indivíduo que circula pelas vias públicas não mais se limita entre a percepção dos quatro planos bidimensionais da calçada, fachada, cobertura e rua. Hoje, soma-se um quinto elemento perceptivo, o do ciberespaço (Ascott, 1995), sob as telas virtuais da navegação digital.

A navegação digital em aplicativos de celulares compõe em um único dispositivo portátil uma gama de informações interativas e atualizadas do ambiente construído, até então impossíveis de serem disponibilizadas em sua totalidade no meio analógico. Dentre os mais variados estilos analógicos, desde mapas históricos em pergaminho, placas e diretórios urbanos e os "guias de ruas" (Garcia, 2017), as informações espaciais, enfim, migraram ao ambiente virtual.

Ao comparar as informações em meio analógico e digital, verifica-se a consistência de um sistema integrado ao pedestre, com um mapa localizador, setas e orientações, identificação de vias e marcos e a distância e tempo dos trajetos. Porém, incorporam-se outros atributos e particularidades do meio tecnológico (Wang et al., 2005), que integram outros filtros de informações necessários para qualificar as caminhadas urbanas (Tabela 1).

Entre os atributos mencionados, destacam-se (1) a interatividade da interface digital com os recursos touch-screen de seleção de objetos ou de aproximação e distanciamento do mapa e detalhamentos; (2) a dinâmica das informações com soluções de realidade aumentada, mapeamento tridimensional, conteúdo multimídia e guias sonoros; (3) a automatização dos trajetos calculados, considerando estimativa da distância percorrida, tempo de percurso e altimetria; (4) a confiabilidade das informações devido a frequente atualização do sistema e o compartilhamento instantâneo de conteúdos entre os usuários; (5) a acessibilidade do meio digital, pelo alcance de dispositivos móveis e o recurso de localização geográfica do Sistema de Posicionamento Global (GPS). 
Tabela 1: Análise comparativa de informação para pedestres em sistemas analógicos e digitais.

\begin{tabular}{lll}
\hline Atributos & Sistemas analógicos & Sistemas digitais \\
\hline $\begin{array}{l}\text { Integridade das } \\
\text { informações }\end{array}$ & $\begin{array}{l}\text { Quantidade limitada de informações } \\
\text { devido à interface fixa e estática. }\end{array}$ & $\begin{array}{l}\text { Amplitude de informações } \\
\text { devido à interface interativa e } \\
\text { dinâmica. }\end{array}$ \\
$\begin{array}{ll}\text { Acessibilidade das } \\
\text { informações }\end{array}$ & $\begin{array}{l}\text { Implementação em pontos } \\
\text { estratégicos da cidade, nem sempre } \\
\text { disponíveis durante o trajeto. }\end{array}$ & $\begin{array}{l}\text { Dispositivos compactos, móveis } \\
\text { durante todo o percurso. }\end{array}$ \\
$\begin{array}{l}\text { Confiabilidade das } \\
\text { informações }\end{array}$ & $\begin{array}{l}\text { Baixa credibilidade devido à falta de } \\
\text { manutenção da infraestrutura fixa e } \\
\text { os atos de vandalismo com danos } \\
\text { materiais. }\end{array}$ & $\begin{array}{l}\text { Alta credibilidade devido à } \\
\text { frequentes atualizações de } \\
\text { sistemas digitais e o } \\
\text { compartilhamento de conteúdo } \\
\text { entre usuários. }\end{array}$ \\
\hline
\end{tabular}

Por um lado, os tradicionais sistemas fixos e analógicos de informação para pedestres oferecem às vias públicas uma identidade visual singular e atendem à demanda do deslocamento ativo na cidade para aqueles que nem sempre têm acesso integral ao online e digital. Ainda assim, apesar de seu papel essencial como infraestrutura urbana, os sistemas analógicos não satisfazem por inteiro os pedestres da contemporaneidade (Wang et al., 2005).

A tecnologia inserida como uma extensão do homem está aprimorando a comunicação e a criação de experiências que conectam pessoas aos seus espaços (Tang, 2011), e oferece uma alternativa mais acessível às informações. Sistemas informativos para pedestres em dispositivos móveis surgem como um complemento que deve agregar valor aos sistemas analógicos já existentes nas cidades (Reising, 2009 apud Jeffrey, 2017).

A conjunção entre o fixo e o digital indica a futura tendência dos sistemas híbridos nas cidades, que consistem em uma rede de displays urbanos informativos customizados, interconectados e integrados com dispositivos móveis (Nordkapp \& Urbanscale, 2011; Society for Experiential Graphic Designs [SEGD], 2021). Soluções como estas evidenciam a aplicabilidade do design de informação diante da nova realidade das cidades digitais, de espaços físicos cada vez mais tecnológicos.

\section{A qualidade das informações espaciais no meio digital}

A tecnologia introduziu o ciberespaço (Lemos, 2010) e a digitalização do mundo real, mas também gerou recursos para a ampla captação e alimentação de dados. O movimento ficou conhecido como Big Data ou dataficação das coisas (Cukier \& Mayer-Schönberger, 2014), e introduziu um novo olhar sob a esfera pública sustentado por um desenvolvimento urbano inteligente. Os dados coletados, quando organizados e armazenados em bancos digitais, geram diferentes associações até então despercebidas, agregando conhecimento e informação de qualidade. 
A cidade passou a ser considerada um laboratório aberto, uma matrix de dados territoriais (Ascott, 1995), e os indivíduos conectados tornaram-se sensores móveis. Geralmente, estes dados são captados por tecnologias sofisticadas, como sensores e câmeras, que registram em tempo real as informações "invisíveis" do espaço, como qualidade do ar e nível de ruído em determinada região (Fab Lab Barcelona, 2018; Mobilidade Curitiba, 2019; Nordkapp \& Urbanscale, 2011). Tais informações podem ser introduzidas nos aplicativos de navegação digital para calcular rotas mais sustentáveis ${ }^{1}$ ou mais atrativas. ${ }^{2}$

Um outro método de captação de dados baseia-se nos dispositivos móveis como fonte de informações. Por exemplo, o rastreamento de sistemas de GPS ativos em celulares possibilita o desenvolvimento de mapas atualizados da densidade urbana ${ }^{3}$, ao mesmo tempo que a interação nas redes sociais permite mapear a cidade de acordo com a qualidade sensorial e perceptiva do espaço construído. ${ }^{4}$

Assim, as inovações tecnológicas combinadas à navegação digital em dispositivos móveis indicam um aprimoramento das camadas de informações, tornando-as mais compatíveis com as necessidades e preferências de cada pedestre. Nos últimos anos, o Google Maps chegou a propor uma melhoria dos trajetos para caminhadas (BBBC News, 2021; Fogaça, 2019), enquanto outros projetos também vêm desenvolvendo mapas urbanos baseados em percursos mais prazerosos ao pedestre de acordo com a qualidade do ambiente construído. ${ }^{5}$

Tudo indica a inclusão de outras variáveis além da distância e tempo para o cálculo de rotas a pé na navegação digital, com camadas de informações mais completas, precisas e atualizadas que visam a qualidade e o estímulo do caminhar urbano. Em um futuro próximo, a usabilidade de aplicativos de navegação digital tende a ser mais eficiente e satisfatória aos pedestres da cidade.

\section{0 processamento das informações espaciais}

As informações espaciais contribuem para a legibilidade e orientação urbana no deslocamento a pé nas cidades. Para que cumpram este papel fundamental, as informações devem ser processadas em três diferentes etapas: a perceptiva, cognitiva e comportamental (Figura 1).

\footnotetext{
${ }^{1}$ PlumeLabs (n.d.). Plume Labs: Air quality app. Disponivel em: Plume Labs.

${ }^{2}$ Likeways (2016). Disponível em Likeways.

${ }^{3}$ MIT Senseable City Lab. (n.d.). Real Time Rome. Disponivel em Real Time Rome.

${ }^{4}$ Goodcitylife.org. (2016). The Good City Life. Disponível em The Good City Life.

${ }^{5}$ MIT Senseable City Lab. (n.d.). Desirable Streets. Disponível em Desirable Streets, MIT Senseable City Lab.
} 
Figura 1: Etapas do processamento de informações espaciais.

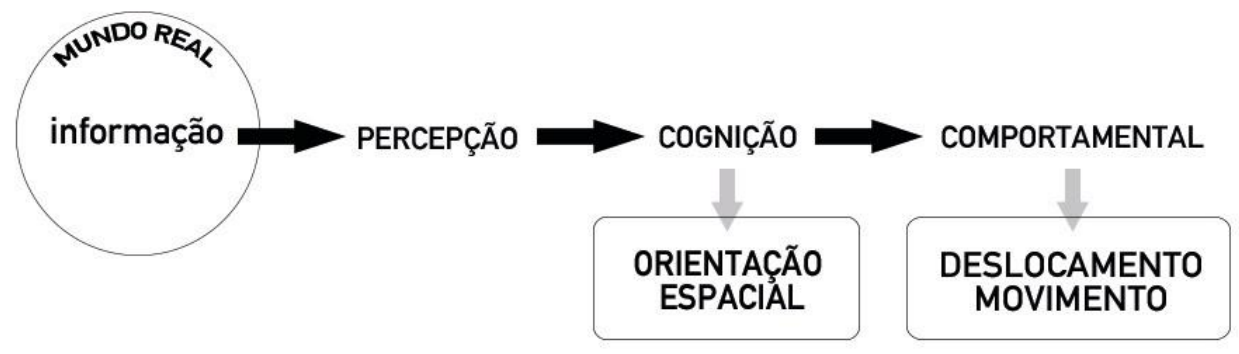

Quando um indivíduo caminha pela rua, ele ativa a sua atenção aos vários estímulos disponíveis no espaço. A atenção é o meio pelo qual se organiza e processa, conscientemente e inconscientemente, um determinado número de informações dentre as tantas disponíveis (Norman, 2006). O nível de alerta de atenção do pedestre corresponde ao seu processo ativo de percepção, referente a captação de determinadas informações via receptores sensoriais, em especial, aqueles relacionados à visão e audição.

A mente, então, recebe estas informações sensoriais percebidas e as manipula, tornandoas em representações mentais, também conhecidas como padrões ou imagens mentais, que contêm um significado. A significação das representações mentais refere-se a compreensão delas, e está associada a um conjunto de emoções, impressões, preferências e crenças diante da experiência perceptiva subjetiva do sujeito. Uma vez compreendidas, as imagens mentais podem ser armazenadas na memória e recuperadas posteriormente. A aprendizagem ocorre quando o indivíduo é capaz de identificar as informações externas captadas diante de experiências e decodificá-las mentalmente, construindo imagens mentais e memorizando-as (Sternberg, 2010).

É a partir deste processo cognitivo que ocorre a orientação espacial do pedestre. Ao caminhar pela cidade e perceber as informações disponíveis ao seu redor, como aquelas inseridas nos aplicativos de navegação digital, o indivíduo as processa mentalmente, criando mapas mentais que o situam no ambiente construído (Locatelli, 2007). Quando bem orientados, os pedestres já reconhecem as informações espaciais, bem definidas em seu mapa mental e armazenadas na memória, e podem se deslocar na cidade de maneira habitual, com um nível comportamental no processamento cerebral (Norman, 2006). Quando ocorre a desorientação espacial, o sujeito tende a processar as informações externas em um nível reflexivo e consciente, com total atenção, para seguir com o processo perceptivo e cognitivo descrito anteriormente.

A orientação espacial facilita o planejamento de trajetos e, em sequência, a tomada de decisão na escolha de apenas uma rota dentre as tantas planejadas. A decisão é, então, transformada em ação com o deslocamento do pedestre na rota previamente calculada. É neste sentido que o autor Passini (1996 apud Locatelli, 2007) define o caminhar como um movimento intencional no espaço. 


\section{O processamento de informações digitais}

Com o celular em mãos, as informações perceptíveis encontram-se essencialmente no meio digital, na interação humano-computador e fora do campo sensorial imediato da pessoa, configurando a então chamada ciber percepção (Ascott, 1995). O homem moderno redefine os seus sentidos perceptivos baseando-se em suas extensões digitais e concebe mapas mentais provenientes da dimensão virtual.

A interface digital adentra cada vez mais o mundo físico, atuando como um mediador cognitivo (Lemos, 2010). O ambiente, agora dinâmico, interativo e conectado, direciona as pessoas a novos caminhos tanto fisicamente como cognitivamente. Ou seja, a forma como as informações são colocadas no meio digital induzem novas maneiras de perceber, compreender, pensar e agir. Por exemplo, as diferentes camadas de informações espaciais nos aplicativos de navegação digital estabelecem categorias hierárquicas que refletem nas categorias cognitivas e, consequentemente, na ordem de planejamento e tomada de decisão (Norman, 2006).

\section{A terceirização da memória}

A navegação digital atua como um suporte ao modelo cognitivo interno, como uma exteriorização da imagem mental (Norman, 2006). Nesse sentido, ao mesmo tempo que o pedestre pode reconhecer as suas limitações de ver, pensar e agir diante da ausência das ferramentas tecnológicas, ele também se aproveita do suporte da navegação digital para melhor se localizar e se locomover no espaço com uma carga cognitiva reduzida (Mondschein \& Moga, 2018).

A facilidade da leitura espacial e o conforto cognitivo diante da qualidade e quantidade de informações no meio digital sugere a terceirização da memória na orientação espacial do pedestre. $O$ fato de desenvolver ferramentas cada vez mais elaboradas no deslocamento a pé nas cidades contribui para ampliar as múltiplas leituras da imagem urbana e os conhecimentos do indivíduo sobre seu território, ao mesmo tempo que consome pouco espaço em sua memória.

Por outro lado, a prevalência dessas ferramentas pode levar a uma redução da capacidade cognitiva de localização. Alguns estudos (Field et al., 2011; Mondschein \& Moga, 2018) indicam uma limitação da memorização de informações e do reconhecimento espacial ao se caminhar com o apoio da navegação digital.

Nesse caso, os recursos tecnológicos tendem a se sobressair diante da memória humana, estabelecendo uma relação de dependência cognitiva. O homem abre mão de seu controle sob as tecnologias, perdendo ou não desenvolvendo habilidades cognitivas para a sua orientação espacial e adquirindo mais confiança nos trajetos indicados pelos aplicativos do que aqueles planejados pelo seu próprio processo mental. A orientação espacial passa a ser vinculada à eficiência das ferramentas digitais (Mondschein \& Moga, 2018), enquanto que o pedestre corre maior risco de desorientação ao desviar da direção calculada nos aplicativos.

A vivência urbana também é diretamente afetada. Dependendo da familiaridade do pedestre com a ferramenta, a velocidade de seu percurso pode ser alterada, com mais ou menos tempo de pausa para conferir a rota no celular, e a sua percepção pode se enquadrar apenas sob as telas digitais, empobrecendo as possibilidades de experiências no espaço urbano. 


\section{Conclusão}

A tendência do aprimoramento das informações espaciais em dispositivos móveis contribui para a mobilidade ativa das grandes cidades. Ao ampliar as capacidades de conhecimento da malha urbana, a navegação digital promove a orientação espacial do pedestre com um processamento cerebral mais confortável, de baixa carga cognitiva e de armazenamento. Por outro lado, elas atuam como modelos mentais exteriorizados, terceirizando a memorização das informações e limitando os processos perceptivos, cognitivos e comportamentais do pedestre. Em futuras investigações, sugere-se um aprofundamento dos modelos mentais diante da digitalização de sistemas informativos. Cabe, enfim, refletir sobre os limites entre as fronteiras do real e do digital, ao desfrutar das aprendizagens fornecidas pelas ferramentas tecnológicas (Tang, 2011) de maneira que elas não se sobressaiam às categorias humanas, em uma constante busca de um consumo mais consciente de uma tecnologia com propósito.

\section{Referências}

Ascott, R. (1994). The architecture of cyberception. Leonardo Electronic Almanac. Disponível em https://www.academia.edu/740566/The_architecture_of_cyberception

BBC News. (2021, 31 de março). Google Maps to start showing eco-friendly routes. Disponível em https://www.bbc.com/news/business-56585877

Cukier, K., \& Mayer-Schönberger, V. (2014). The rise of Big Data: How it's changing the way we think about the world. Foreign Affairs, 92(3), pp.28-40.

https://doi.org/10.1515/9781400865307-003

Fab Lab Barcelona. (2018, 7 de dezembro). Documentary: Citizen Science Revolution - Making Sense [Video]. Youtube. Disponível em https://www.youtube.com/watch?v=hvn5LyACUYw\&t=2810s

Field, K., O'Brien, J., \& Beale, L. (2011). Paper maps or GPS? Exploring differences in wayfinding behaviour and spatial knowledge acquisition. In Proceedings of the 25th International Cartographic Conference, pp.1-8. Europa Organisation.

Fogaça, A. (2019). Google Maps prepara recurso para indicar ruas mais iluminadas. TecnoBlog. Aplicativos de Software. Disponível em https://tecnoblog.net/317189/googlemaps-indicar-ruas-iluminadas/

Garcia, R. (2017, 14 de junho). A vida sem Waze: os antigos guias de rua de São Paulo. Veja São Paulo. Seção Cidades. Disponível em: https://vejasp.abril.com.br/blog/memoria/a-vidasem-waze/

Hall, E. T. (2005). A dimensão oculta. São Paulo: Editora Martins Fontes.

Jeffrey, C. (2017). Wayfinding Perspectives: Static and digital wayfinding systems - can a wayfinding symbiosis be achieved? In A. Black, P. Luna, O. Lund, \& S. Walker (Ed.), Information Design: Research and Practice, pp. 509-526. Routledge.

Lemos, A. (2010). Celulares, funções pós-midiáticas, cidade e mobilidade. Revista Brasileira de Gestão Urbana, 2(2), pp.155-166. Disponível em https://www.redalyc.org/articulo.oa?id=193115578002 
Locatelli, L. (2007). Orientação espacial e características urbanas. [Dissertação Mestrado em Planejamento Urbano e Regional]. Faculdade de Arquitetura e Urbanismo, Universidade Federal do Rio Grande do Sul, Porto Alegre.

Mobilidade Curitiba. (2019, 30 de agosto). Placas de rua de Curitiba terão estrutura para tecnologia. Disponível em https://mobilidadecuritiba.com.br/estrutura-para-tecnologia

Mondschein, A., \& Moga, S. T. (2018). New Directions in Cognitive-Environmental Research. Journal of the American Planning Association, 84:3-4, pp. 263-275. https://doi.org/10.1080/01944363.2018.1526644

Nordkapp \& Urbanscale. (2011). Urbanflow Helsinki: building an operating system for everyday life. Disponível em http://helsinki.urbanflow

Norman, D. (2006). O design do dia-a-dia. Tradução de Ana Deiró. Rio de Janeiro: Rocco.

Society for Experiential Graphic Designs. (n.d.). LinkNYC - a Step Toward Smart Cities? Disponível em https://segd.org/smart-cities

Society for Experiential Graphic Designs. (n.d.). Interconnect with CityID. Disponível em https://segd.org/interconnect-city-id

Sternberg, R. (2010). Psicologia Cognitiva. Tradução da 5 ed. norte-americana. São Paulo: Cengage Learning.

Tang, L. (2011). In search of an architectural legibility: Human Movement behavior and Wayfinding for Pattern Design. [Dissertação Mestrado]. Faculdade de Arquitetura, Universidade de Cincinnati, Cincinnati.

Wang,Y., Hedgecock, E. M., \& Fernandez,O. (2005). Mobile media and digital wayfinding: Strategies for implementation. Disponível em http://www.units.muohio.edu/

\section{Sobre as autoras}

Aline de Camargo Barros, Ma., UDESC, Brasil <alinecamargobarros@gmail.com> Gabriela Botelho Mager, Dra., UDESC, Brasil < gabriela.mager@udesc.br> 